Short communication

\title{
Peak crossover in high-performance liquid chromatography elution monitored using whole-column detection
}

\author{
Shu-Hui Lin ${ }^{a}$, Tiing Yu ${ }^{\mathrm{a}, *}$, Alf Sheu ${ }^{\mathrm{b}}$, Der-Jyh Yang ${ }^{\mathrm{b}}$, Su-Cheng Pai ${ }^{\mathrm{c}}$ \\ a Department of Applied Chemistry, National Chiao Tung University, Hsinchu 30050, Taiwan \\ ${ }^{\mathrm{b}}$ Avision Inc., 20 Creation Road, Science-Based Industrial Park, Hsinchu, Taiwan \\ ${ }^{c}$ Division of Marine Chemistry, Institute of Oceanography, National Taiwan University, Taipei, Taiwan
}

\section{A R T I C L E I N F O}

\section{Article history:}

Received 28 March 2008

Received in revised form 9 June 2008

Accepted 12 June 2008

Available online 18 June 2008

\section{Keywords:}

Peak crossover

Retention-order inversion

Whole-column detection

Multi-channel detection

HPLC

\begin{abstract}
A B S T R A C T
A whole-column detection (WCD) system was fabricated using the parts from a typical A4 size optical scanner. Optimum layout of the fluorescence tube, beam condenser and photon transducers from the scanner made an effective devise to examine the dynamic chromatographic separation process. This system was applied in monitoring peak crossover in an ion-pair chromatographic elution. A stage-wise gradient was conducted in eluting a mixture of New Coccine and Carmoisine. Compound New Coccine lagging behind after sample injection moved passing Carmoisine at the column outlet. The complete on-column elution process could be observed directly using this detection system. This inexpensive and rugged WCD system provided a $0.3 \mathrm{~mm}$ spatial resolution and a $3.6 \mathrm{~ms}$ temporal resolution and was proven to be an adequate device to directly monitor solute retention behavior in the liquid chromatography column.
\end{abstract}

(c) 2008 Elsevier B.V. All rights reserved.

\section{Introduction}

High-performance liquid chromatography (HPLC) is one of the most powerful tools for modern chemical analysis. Usually, singlepoint detection is carried out by a post-column detector in HPLC. However, some chromatographers considered that single-point detector may not be the only one detection method for LC. The concept of whole-column detection (WCD) [1] was first proposed by Gelderloos et al. in a theoretical study of the peak distribution along a chromatographic column. In the WCD simulation, the authors demonstrated that on-column peaks were resolved with a higher resolution in less time than off-column peaks obtained on the chromatogram in longer time. The WCD chromatography might provide better ability to predict the elution behavior. In addition, the authors suggested a crossover of solute peaks could occur during a gradient elution using computer simulation, that is, a solute moving slower than another solute after sample injection migrates faster afterwards and reaches the column outlet earlier.

The crossover of solute peaks cannot be detected directly by the conventional single-point detection method because the postcolumn detector can only provide the outcome off the column but not what has happened on-column. An on-column WCD system was required to monitor the peak crossover. Various WCD systems

\footnotetext{
* Corresponding author. Tel.: +8863573 1673; fax: +88635723764.

E-mail address: tyu@faculty.nctu.edu.tw (T. Yu).
}

have been fabricated for observing on-column process. Rowlen et al. designed the first WCD instrument [2,3]. Eleven discrete sources and photodiodes were evenly installed on a glass column. Evans and McGuffin [4] used two-point detection on an open capillary in order to isolate extra-column variance. Wang and Hartwick designed a moving column detection method in capillary isoelectric focusing system [5]. Tamura et al. [6] fabricated a fluorescence imaging system for monitoring the entire column. Wu et al. [7] reviewed the development in WCD, including partial column imaging detection and whole-column imaging detection for HPLC and capillary electrophoresis. The analysis speed would be accelerated for some applications due to the capability of direct monitoring the oncolumn separation process $[8,9]$.

We fabricated a WCD system using the parts taken from a common optical scanner. This laboratory-made WCD allowed an observation range of $21 \mathrm{~cm}$ that was wide enough to cover complete solute peaks during the elution. In addition, a high-pressure glass column was assembled and packed with reverse-phase stationary phase. The combination of the column and the WCD system allowed us to monitor the whole solute peak signals during the elution under typical HPLC conditions.

Using ion-pair HPLC for determining synthetic dyes, Kiseleva et al. [10] found that the retention order of a pair of dye, that is, New Coccine and Carmoisine, was inverted in two separate chromatographic runs. The inversion was highly dependent on the concentration of the ion-pair reagent and the concentration of the buffer solution in the mobile phase. The retention time change 


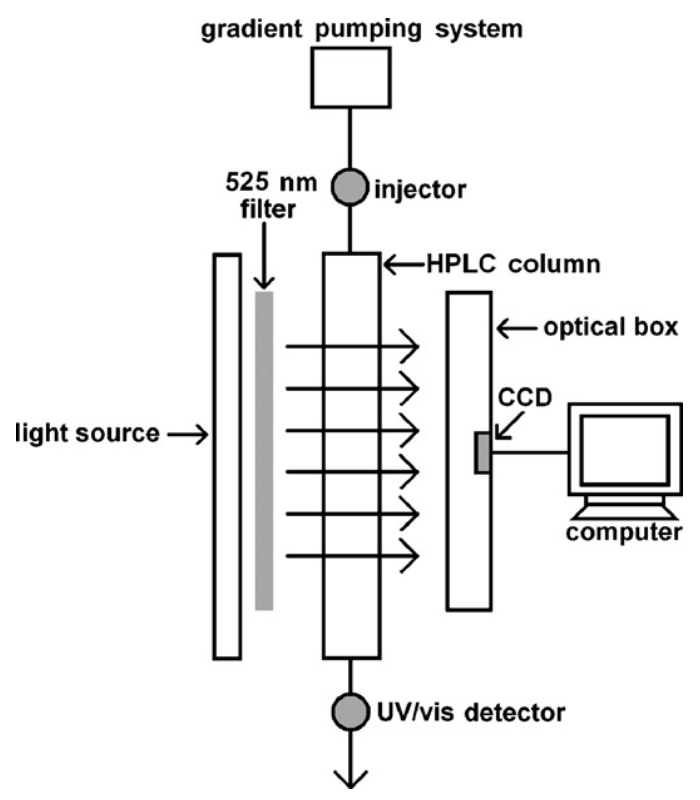

Fig. 1. Schematic diagram of the experimental system.

seemed to be very pronounced especially for the New Coccine molecules. This sample mixture appeared a good candidate for observing peak crossover in an elution. We demonstrated this phenomenon with the dye mixture using the laboratory-made WCD system.

\section{Experimental}

\subsection{High-pressure glass column}

The experimental set-up, shown in Fig. 1, was composed of a gradient pumping system (Series 4, Lab Alliance, PA, USA), a Valco six-way sample injector with a $20 \mu \mathrm{L}$ injection loop, the laboratorymade HPLC column and WCD system, and a Waters 486 (Milford, MA, USA) UV/vis detector installed at the outlet of the column for obtaining conventional chromatograms. In order to accommodate typical HPLC experimental conditions, we assembled a high-pressure column with a glass window to allow absorbance measurement inside the column. As shown in Fig. 2, an $18 \mathrm{~cm}$ long acid/alkali resistant glass tube ( $3 \mathrm{~mm}$ I.D. $\times 8 \mathrm{~mm}$ O.D.) was contained in a $20 \mathrm{~cm}$ long stainless steel pipe ( $8 \mathrm{~mm}$ I.D. $\times 20 \mathrm{~mm}$ O.D.). Two cuts $(15 \mathrm{~cm} \times 4 \mathrm{~mm})$ were made on the stainless steel pipe to create a light path for absorbance measurement. Two male nuts were connected to the stainless steel pipe and the high pressure was held inside the glass tube with viton $o$-rings $(4.47 \mathrm{~mm} \times 1.78 \mathrm{~mm})$. A Swagelok female fitting $(0.635 \mathrm{~cm})$ was then attached to the tubing $(0.635 \mathrm{~cm})$ on the male nut at each side of the column. An external column end connector, equipped with a frit $(0.45 \mu \mathrm{m})$, was linked

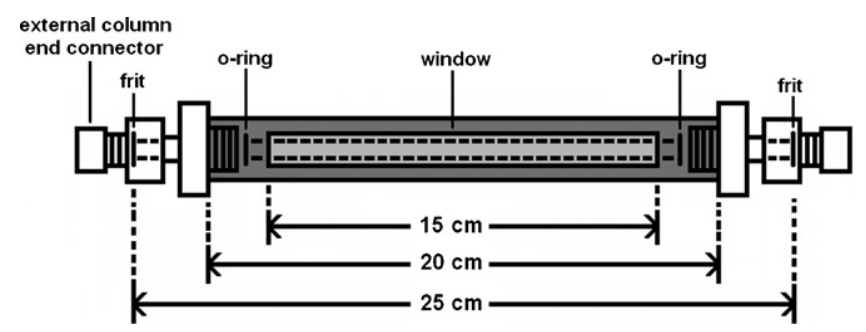

Fig. 2. Schematic diagram of the HPLC column with a $15 \mathrm{~cm}$ absorbance measurement window. to the Swagelok female fitting on each side. This column was then packed with $10 \mu \mathrm{m}$ octadecylsilane stationary phase. The stationary phase was packed in the space between the two frits, so column position was measured starting from the inlet frit. The earliest position for the solute signals to appear began with $5 \mathrm{~cm}$ from the frit. The column could be easily connected to the injection valve with a male zero-dead-volume nut. The other side of the column was linked with the UV/vis detector. The pressure limit for this glass column is $200 \mathrm{~kg} \mathrm{~cm}^{-2}$ and the highest pressure operated in this study was $93 \mathrm{~kg} \mathrm{~cm}^{-2}$. The column was installed on a translational stage for whole-column detection.

\subsection{WCD system}

Most parts of the WCD system were taken from an A4 size scanner (CanoScan 5000, Avision Inc., Hsinchu, Taiwan). The optical scanner was composed of a scanning optical cartridge, a glass platform to hold up the documents, and an electronic interface to transmit digital images to a computer. The scanning optical cartridge was made from a light tube (a cold cathode fluorescent lamp, $21 \mathrm{~cm}$ long $\times 2 \mathrm{~mm}$ O.D.) and an optical box (including three mirrors and one lens) which could condense the image of a $21 \mathrm{~cm}$ long object onto four pairs (two for each pair) of charge-coupled device (CCD) transducers. The CCD transducers were all Toshiba models T8E21. The coatings on the three pairs of CCD allowed green, red, and blue light to pass, respectively; meanwhile, the fourth pair (also called "grey") remained uncoated. The scanning optical cartridge and the electronic interface were taken apart from the scanner case. The light tube was mounted on a translational stage, and a $21 \mathrm{~cm} \times 2 \mathrm{~cm}$ interference filter (band center $=525 \mathrm{~nm}$, bandwidth $=70 \mathrm{~nm}$, transmittance $=95 \%$ ) was installed close to the light source. Since the absorbance maxima of New Coccine and Carmoisine were 506 and $515 \mathrm{~nm}$, respectively; this filter ensured a narrow bandwidth of the source for the absorbance measurements. Alignment was adjusted using the translational stage to allow the source radiation to pass through the filter, and the column, and then enter the optical box to give a linear image unto the CCD. The data fetched only by the grey CCD (better sensitivity than those with coatings) pair were transmitted to a computer for further analysis.

There were 5360 pixels on each CCD. Each exposure thus gave 10,720 data points of radiant power that covered the $21 \mathrm{~cm}$ long image. The data points, however, could be handled in a way that accumulated the total photon counts of the adjacent pixels. Accordingly, it was possible to increase the detection sensitivity and decrease the total data points to be transmitted to the computer for analysis. In this work, the total photon counts of 16 adjacent pixels were combined to make one point of data. This operation therefore generated 670 data points for each exposure on the CCD, and gave a $\sim 0.3 \mathrm{~mm}(21 \mathrm{~cm} / 670)$ spatial resolution for the signals. Since the window of the high-pressure glass column was $15 \mathrm{~cm}$, the effective data points on the CCD were $478(15 \mathrm{~cm} / \mathrm{spatial}$ resolution). With a very short exposure period $(3.6 \mathrm{~ms})$ on the $C C D$, the moving peaks were considered "frozen" during the consecutive "snap shots."

Although the packing material is not transparent, scattering occurred between the interstices of the packing material, and the diffuse-transmitting light would eventually reach the CCDs mounted at the other side of the column. The radiation from the light tube of the WCD was first recorded as the background radiant power $\left(P_{0}\right)$, while the column was eluted with pure mobile phase under the desired flow rate. After sample injection, the transmitted radiant power $(P)$ was measured again during the elution. The measurement could be treated as absorbance of sample peak profile $[2,3]$ in the column and was calculated using the following formula: $A=\log \left(P_{0} / P\right)$. The acquired signal resulted from the total absorbance of analyte molecules on the stationary and mobile phases with 
different sensitivities [3]. In this study, peak areas of different retention capacities remained in only $0.07 \%$ difference. Accordingly, the real-time absorbance signals represented the sample spatial profile inside the column very well [6,8]. In the meantime, chromatograms were acquired using the off-column UV/vis detector.

\subsection{Reagents and chemicals}

Sample solutions of New Coccine $(0.50 \mathrm{mM})$ and Carmoisine $(0.60 \mathrm{mM})$, purchased from Aldrich (WI, USA), and a mixture of these two compounds $(0.17 \mathrm{mM}$ for New Coccine and $0.20 \mathrm{mM}$ for Carmoisine) were all prepared in de-ionized water. Elution solvent and other chemicals including acetonitirile (ACN), sodium dihydrogen phosphate $\left(\mathrm{NaH}_{2} \mathrm{PO}_{4}\right)$, phosphoric acid $\left(\mathrm{H}_{3} \mathrm{PO}_{4}\right)$, and tetrabutylammonium dihydrophosphate (TBA) were all highpurity grades and were obtained from Aldrich.

\section{Results and discussion}

Peak inversion of the samples in separate runs could be realized by changing phosphate and the TBA concentration of the mobile phase [10]. Unfortunately the baseline of the solute peaks fluctuated with the concentration change of the TBA. We then carried out crossover examination by changing the phosphate concentration only. In order to achieve peak crossover using gradient elution, we first tried to find two mobile phases that would probably cause peak inversion in two separate isocratic elutions. Mobile phase A (40\% ACN in a pH 4.2, $50 \mathrm{mM}$ phosphate buffer, containing $10 \mathrm{mM}$ TBA) and mobile phase $\mathrm{B}(40 \% \mathrm{ACN}$ in a $\mathrm{pH} 4.2,5 \mathrm{mM}$ phosphate buffer, containing $10 \mathrm{mM}$ TBA) were found able to attain this phenomenon (as shown in Fig. 3). Accordingly, several gradient elutions were performed in order to look for the possible crossover. However, distinct crossover did not occur no matter what slope of the changing rate was from mobile phase $B$ to mobile phase A.

We then decided to apply a stage-wise gradient elution to change the solvent strength more quickly than the continuous gradients. The samples of the two individual compounds were first eluted using mobile phase B for $4 \mathrm{~min}$ and were switched to mobile phase A. At the beginning, the individual solute migration speed was relatively slow due to the weaker solvent strength of mobile phase B. After switching to mobile phase A, both solutes moved faster. The on-column peak profiles for the individual compounds are shown in Fig. 4(a). The profiles on the spatial coordinate were

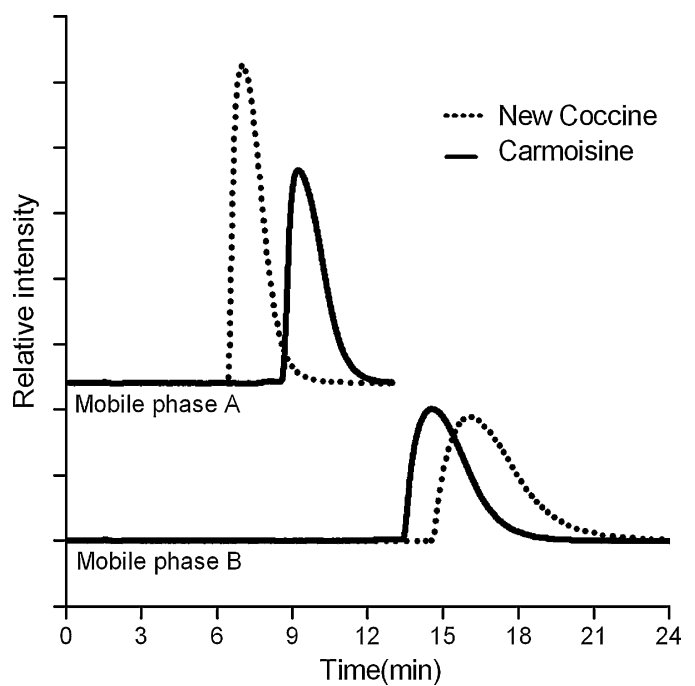

Fig. 3. Chromatograms of dyes eluted with mobile phase $A(40 \% A C N$ in a $p H 4.2$, $50 \mathrm{mM}$ phosphate buffer, containing $10 \mathrm{mM}$ TBA) and mobile phase B (40\% ACN in a $\mathrm{pH} 4.2,5 \mathrm{mM}$ phosphate buffer, containing $10 \mathrm{mM} \mathrm{TBA}$ ); flow rates: $1 \mathrm{ml} / \mathrm{min}$. Notice that four separate runs were conducted, and the chromatograms using the same mobile phase were plotted together.

recorded while the elution times were 4.6, 5.1, 5.6, 6.1, 6.6, 7.1, and $7.6 \mathrm{~min}$, respectively. As time elapsed, the profile became broader with lower peak height as expected. It is noted that the distances between the peaks became greater after $5.1 \mathrm{~min}$. The last 4 gaps remained essentially constant due to the same solute migration rate after switching to mobile phase A. A solute migration map (Fig. 4(b)) was constructed by locating the moving solute peaks according to their positions on the column and the monitoring time. The solute migration rate was equal to the slope of the curve in the migration map. The migration rate for both compounds started to accelerate at $\sim 5.1 \mathrm{~min}$, illustrated by the larger slopes. A $1.1 \mathrm{~min}$ delay for the mobile phase A to catch up the solute was observed. As can be seen, there is a cross point (elution time at $\sim 6.1 \mathrm{~min}$ and column location at $\sim 12.4 \mathrm{~cm}$ ) on the map; that suggested the crossover could occur for the mixture of these two compounds. The steeper slope for compound New Coccine reflected a relatively faster speed with mobile phase A than that of Carmoisine. A mixture of the two compounds was then investigated. As shown in Fig. 5(a), a peak with one appar-
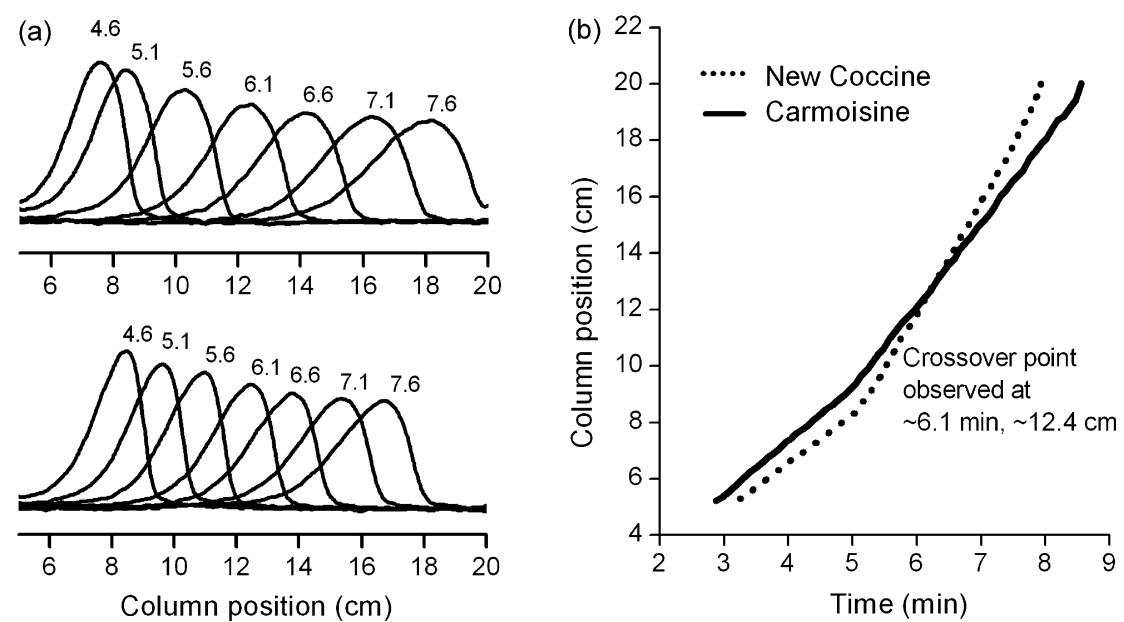

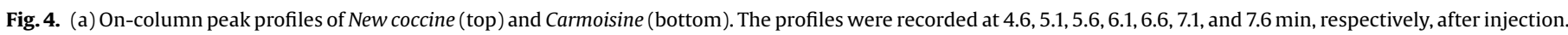

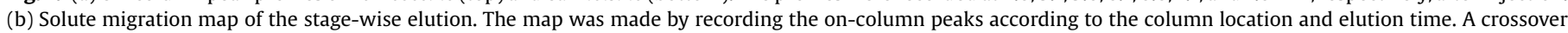
was observed at $\sim 6.1 \mathrm{~min}$ and $\sim 12.4 \mathrm{~cm}$. 

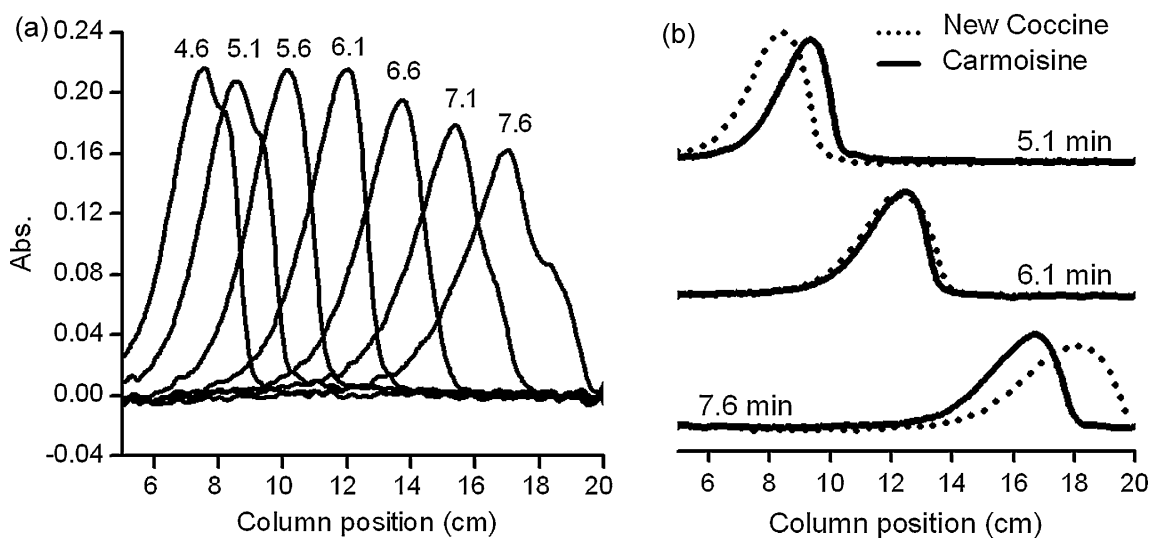

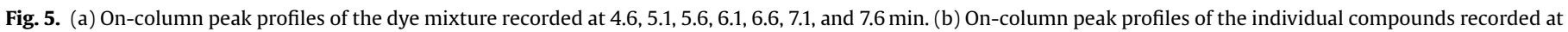
5.1, 6.1, and $7.6 \mathrm{~min}$.

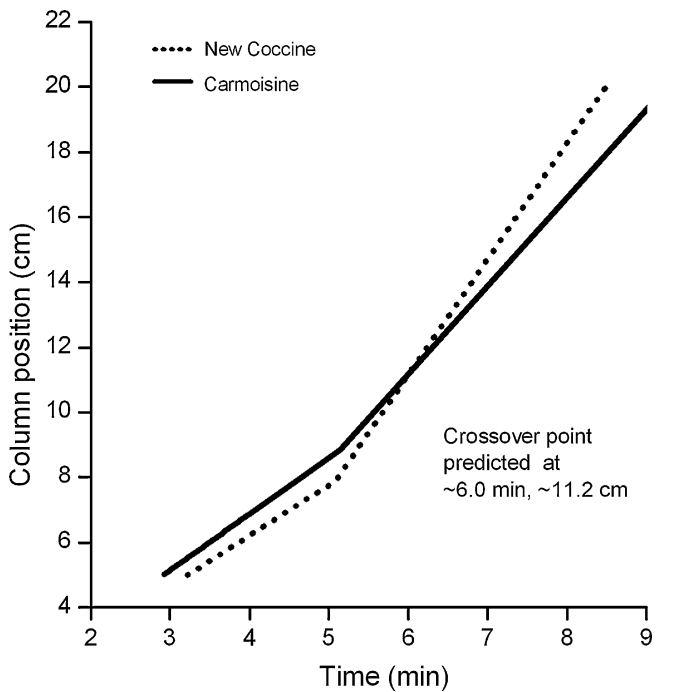

Fig. 6. The solute migration map calculated using Eq. (1) with post-column detection data. A crossover was predicted at $\sim 6.0 \mathrm{~min}$ and $\sim 11.2 \mathrm{~cm}$.

ent shoulder appeared after injection. This peak then moved faster and merged with the shoulder to form one peak. As time elapsed, the moving peak split into another peak with an apparent shoulder. Fig. 5(b) shows the overlay plot of the peak profiles of individual runs at 5.1, 6.1, and 7.6 min. Compound New Coccine lagged Carmoisine at $5.1 \mathrm{~min}$, while mobile phase A just moved close to the solutes. After mobile phase A took effect, New Coccine moved faster and came together with Carmoisine right at $6.1 \mathrm{~min}$, and apparently passed Carmoisine at 7.3 min. Qualitatively, Fig. 5(b) matched the spatial peak profiles shown in Fig. 5(a) very well. It thus confirmed the peak crossover.

The relationship between the migration time and migration distance in a stage-wise gradient elution could also be predicted using the following equation with chromatographic data obtained by the post-column detector [11,12]:

$t_{\mathrm{R}}=\frac{L}{K_{2}}+\frac{u\left(K_{2}-K_{1}\right)}{u K_{2}-K_{1}} t_{\mathrm{c}}-V_{0}$

where $t_{\mathrm{R}}$ was the elution time, $L$ peak location in the column, $u$ the linear flow rate of the mobile phase, $t_{c}$ time of solvent change, $V_{0}$ the column void volume, $K_{1}$ and $K_{2}$ the apparent migration rates of the first and second solutes, respectively. Given different $L$ 's, the equation could be used to construct a solute migration map, as shown in Fig. 6. The migration rates for New Coccine and Carmoisine changed steeply at 5.08 and $5.14 \mathrm{~min}$ and a cross point was predicted at $\sim 6.0 \mathrm{~min}$ and $\sim 11.2 \mathrm{~cm}$. The shape of the simulated curves and the position of cross point were somewhat different from our experimental results, as shown in Fig. 4(b). The earlier occurrence of the peak retention time as well as column location compared with the WCD measurements might take place due to the time delay for mobile phase A to reach to the locations where the peaks were. In other words, Eq. (1) actually assumed an instant change of the mobile phase without any time delay. It still demonstrated the capability to give a very close prediction of peak crossover. However, on-column real-time spatial peak profiles obtained by WCD measurements give more dynamic information in the chromatographic elution.

In conclusion, we fabricated a WCD system in this study to successfully observe the complete peak crossover process in a HPLC gradient elution. This inexpensive and rugged system, composed of parts taken from a typical commercial optical scanner, provided high spatial $(0.3 \mathrm{~mm})$ and temporal $(3.6 \mathrm{~ms})$ resolutions and a wide observation range $(21 \mathrm{~cm})$ for direct HPLC on-column retention data.

\section{Acknowledgement}

Financial support (NSC-95-2113-M-009-031) from the National Science Council of Taiwan is greatly appreciated.

\section{References}

[1] D.G. Gelderloos, K.L. Rowlen, J.W. Birks, Anal. Chem. 58 (1986) 900

2] K.L. Rowlen, K.A. Duell, J.P. Avery, J.W. Birks, Anal. Chem. 61 (1989) 2624

[3] K.L. Rowlen, K.A. Duell, J.P. Avery, J.W. Birks, Anal. Chem. 63 (1991) 575.

[4] C.E. Evans, V.L. McGuffin, Anal. Chem. 60 (1988) 573.

[5] T. Wang, R.A. Hartwick, Anal. Chem. 64 (1992) 1745.

[6] A. Tamura, M. Khademizadeh, K. Ozawa, T. Masujima, Anal. Chim. Acta. 365 (1998) 115.

[7] X.Z. Wu, T. Huang, Z. Lin, J. Pawliszyn, Trends Anal. Chem. 24 (2005) 369

[8] R.A. Shalliker, B.S. Broyles, G. Guiochon, Anal. Chem. 72 (2000) 323.

[9] B. Yao, H. Yang, Q. Liang, G. Luo, L. Wang, K. Ren, Y. Gao, Y. Wang, Y. Qiu, Anal. Chem. 78 (2006) 5845.

[10] M.G. Kiseleva, V.V. Pimenova, K.I. Eller, J. Anal. Chem. 58 (2003) 685

[11] A. Tamura, K. Tamura, S. Razee, T. Masujima, Anal. Chem. 68 (1996) 4000

[12] A. Tamura, M. Khademizadeh, K. Tamura, T. Masujima, J. Pharm. Biomed. Anal. 15 (1997) 1477 